Research Paper

\title{
CHDIL promotes EOC cell invasiveness and metastasis via the regulation of METAP2
}

\author{
Wei-Peng $\mathrm{He}^{1^{*}}$, Yun-Yun Guo ${ }^{*}$, Gui-Ping Yang ${ }^{1 *}$, Hui-Ling Lai ${ }^{1}$, Ting-Ting Sun ${ }^{1}$, Zu -Wei Zhang1, \\ Ling-Long Ouyang ${ }^{1}$, Yu Zheng ${ }^{1}$, Li-Ming Tian ${ }^{1}$, Xiao-Hui Li ${ }^{1}$, Ze-Shan You ${ }^{1}$, Dan Xie ${ }^{2}$ and Guo-Fen \\ Yang 1 四 \\ 1. Department of Gynecology, the First Affiliated Hospital, Sun Yat-Sen University, No. 58, Zhongshan Road II, 510080 Guangzhou, China. \\ 2. State Key Laboratory of Oncology in South China, Cancer Center, Sun Yat-Sen University, No. 651, Dongfeng Road East, 510060 Guangzhou, China. \\ *These authors contributed equally to this work. \\ $\square$ Corresponding authors: Guo-Fen Yang, Department of Gynecology, the First Affiliated Hospital, Sun Yat-Sen University, No. 58, Zhongshan Road II, 510080 \\ Guangzhou, China. E-mail addresses: pgf_yang@126.com; or, Dan Xie, State Key Laboratory of Oncology in South China, Cancer Center, Sun Yat-Sen \\ University, No. 651, Dongfeng Road East, 510060 Guangzhou, China. E-mail addresses: xied@mail.sysu.edu.cn. \\ (c) The author(s). This is an open access article distributed under the terms of the Creative Commons Attribution License (https://creativecommons.org/licenses/by/4.0/). \\ See http://ivyspring.com/terms for full terms and conditions.
}

Received: 2020.05.24; Accepted: 2020.08.16; Published: 2020.08.29

\begin{abstract}
Chromodomain helicase DNA binding protein 1-like (CHDIL) gene has been proposed to play an oncogenic role in human hepatocellular carcinoma. Previously we reported that CHDIL overexpression is significantly associated with the metastasis proceeding of epithelial ovarian cancer (EOC), and may predict a poor prognosis in EOC patients. However, the potential oncogenic mechanisms by which CHDIL acts in EOC remain unclear. To elucidate the oncogenic function of CHDIL, we carried out a series of in vitro assays, with effects of CHDIL ectogenic overexpression and silencing being determined in EOC cell lines (HO8910, A2780 and ES2). Real-time PCR and Western blotting analyses were used to identify potential downstream targets of CHDIL in the process of EOC invasion and metastasis. In ovarian carcinoma HO8910 cell lines, ectopic overexpression of CHDIL substantially induced the invasive and metastasis ability of the cancer cells in vitro. In contrast, knockdown of CHDIL using shRNA inhibited cell invasion in vitro in ovarian carcinoma A2780 and ES2 cell lines. We also demonstrated that methionyl aminopeptidase 2 (METAP2) was a downstream target of CHDIL in EOC, and we found a significant, positive correlation between the expression of CHDIL and METAP2 in EOC tissues $(\mathrm{P}<0.05)$. Our findings indicate that CHDIL plays a potential role in the inducement of EOC cancer cell invasion and/or metastasis via the regulation of METAP2 expression and suggests that CHDIL inhibition may provide a potential target for therapeutic intervention in human EOC.
\end{abstract}

Key words: Ovarian cancer, CHD1L, invasion, metastasis, METAP2

\section{Introduction}

Ovarian cancer is a leading type of cancer among cancers afflicting the female reproductive system and it is the leading cause of death among all gynecological malignancies [1]. The most common pathological type of ovarian cancer is epithelial ovarian cancer (EOC), and it accounts for $95 \%$ of all cases of ovarian cancer. Because EOC is not often accompanied by noticeable symptoms, most patients are not diagnosed until an advanced stage (FIGO III or IV), resulting in a devastating overall 5-year survival rate of around 30\% [2]. Due to the limiting efficacy of chemotherapy, satisfactory tumor cell subtraction is still the most effective treatment for EOC [3], with the greatest challenge arising from extensive metastasis of the pelvic cavity. In addition, metastasis to distal regions of the body outside of the reproductive system is still considered a major factor in the recurrence and possible death in EOC patients [4].

It has been well established that the occurrence and development of EOC is a complex process involving multiple genetic changes, all with multiple 
steps [5]. Therefore, it is a pressing problem to discover and identify the molecular mechanisms of the genes involved in pelvic and peritoneal metastasis in order to improve EOC treatment efficiency and prognosis.

CHD1L (chromodomain helicase DNA binding protein 1-like), also called ALC1 (amplified in liver cancer 1), is a putative oncogene possibly involved in EOC located at chromosome 1q21. The mRNA of CHD1L is 3036 bp long, resulting in a CHD1L protein 897 amino acids in length. The CHD1L protein belongs to SNF2-like family and contains a SNF2-N domain and a HELICs (helicase superfamily c-terminal domain) structure. Most members of the SNF2-like family participate in various nuclear activities, such as transcriptional activation or repression, DNA repair, and recombination. CHD1L is named so due to it being $59 \%$ homologous to CHD1 (chromodomain helicase DNA binding protein 1) which is responsible for modifying chromatin structure [6-8]. Previous work has shown that CHD1L is overexpressed in a variety of human malignancies, including colorectal cancer, breast cancer, gastric cancer, nasopharyngeal cancer, lung cancer, pancreatic cancer, esophageal carcinoma and ovarian cancer, and the degree of CHD1L overexpression is correlated with tumor progression and poor prognosis [9-16]. Previously we reported CHD1L overexpression is significantly correlated with the metastasis proceeding of EOC, and may also be an accurate predictor of poor prognosis in EOC patients [16], suggesting that CHD1L may participate in tumor metastasis. Although CHD1L expression has been extensively characterized in human cancers, the molecular mechanisms underlying aberrant CHD1L expression induced in human malignancies remain unknown.

In order to illuminate the possible roles of CHD1L in the development of EOC, here we investigate the tumorigenicity of CHD1L and the potential molecular mechanisms involved in the invasion and metastasis during EOC, using EOC cell lines. Our results might shed light into the molecular mechanisms of CHD1L involvement in the development, progression and metastasis of EOC.

\section{Results}

\section{Expression of CHDIL in a large cohort of ovarian tissues and its correlation with the clinicopathologic features and survival of EOC patients}

Using a large cohort of clinical EOC tissue, borderline tumors, cystadenomas tissue, and normal ovarian tissue for control, the expression dynamics of
CHD1L were evaluated using immunohistochemistry (IHC). Using previously described protein expression criteria [16], we found that overexpression of CHD1L was observed in $53 \%, 13 \%$ and $6 \%$ of specimens of EOC, borderline tumors, and cystadenomas, respectively, with expectedly no overexpression observed in any normal ovarian tissue sample $(P<0.01$, Table 1, Fig. 1A-C). Upon further analysis, we found that overexpression of CHD1L was significantly, positively correlated with histological type and advanced $\mathrm{pT} / \mathrm{pN} / \mathrm{pM}$ status, FIGO stage of ovarian carcinomas $(P<0.05$, Table 2$)$, and poor survival rates in EOC patients $(P<0.001$, Fig. 1D).

Table 1. The expression of CHDIL in normal ovaries and in benign and malignant epithelial ovarian tumors ${ }^{\mathrm{a}}$

\begin{tabular}{lccl}
\hline & All cases & CHD1L protein \\
\cline { 3 - 4 } & & Normal expression & Overexpression \\
\hline Normal ovaries & 30 & $30(100 \%)$ & $0(0)$ \\
Cystadenomas & 35 & $33(94 \%)$ & $2(6 \%)$ \\
Borderline tumors & 40 & $35(87 \%)$ & $5(13 \%)$ \\
Invasive carcinomas & 160 & $75(47 \%)$ & $85(53 \%)$ \\
\hline a Values are n (\%). A significant increasing frequency of intensive expression of \\
CHD1L was observed in cystadenomas, in borderline tumors and in invasive \\
carcinomas $(P<0.01$, Chi-Square Test for Trend).
\end{tabular}

Table 2. Association of CHDIL expression with patient's clinico-pathological features in 160 ovarian carcinomas

\begin{tabular}{|c|c|c|c|c|}
\hline & \multirow{2}{*}{$\begin{array}{l}\text { All } \\
\text { cases }\end{array}$} & \multicolumn{3}{|c|}{ CHD1L protein } \\
\hline & & $\begin{array}{l}\text { Normal } \\
\text { expression }\end{array}$ & Overexpression & $P$ value a \\
\hline Age at surgery (years) & & & & 0.620 \\
\hline$<51.0 \mathrm{~b}$ & 82 & $40(49 \%)$ & $42(51 \%)$ & \\
\hline$\geq 51.0$ & 78 & $35(45 \%)$ & $43(55 \%)$ & \\
\hline Histological type & & & & 0.024 \\
\hline Serous & 106 & $43(41 \%)$ & $63(59 \%)$ & \\
\hline Mucinous & 19 & $14(74 \%)$ & $5(26 \%)$ & \\
\hline Others ${ }^{c}$ & 35 & $18(51 \%)$ & $17(49 \%)$ & \\
\hline Histological grade (Silv & eberg) & & & 0.142 \\
\hline G1 & 28 & $16(57 \%)$ & $12(43 \%)$ & \\
\hline G2 & 89 & $44(49 \%)$ & $45(51 \%)$ & \\
\hline G3 & 43 & $15(35 \%)$ & $28(65 \%)$ & \\
\hline pT status & & & & $<0.001$ \\
\hline pT1 & 38 & $29(76 \%)$ & $9(24 \%)$ & \\
\hline pT2 & 35 & $15(43 \%)$ & $20(57 \%)$ & \\
\hline pT3 & 87 & $31(36 \%)$ & $56(64 \%)$ & \\
\hline pN status & & & & $<0.001$ \\
\hline $\mathrm{pN} 0$ & 77 & $50(65 \%)$ & $27(35 \%)$ & \\
\hline $\mathrm{pN} 1$ & 83 & $25(30 \%)$ & $58(70 \%)$ & \\
\hline pM status & & & & 0.013 \\
\hline $\mathrm{pMX}$ & 135 & $69(51 \%)$ & $66(49 \%)$ & \\
\hline pM1 & 25 & $6(24 \%)$ & $19(76 \%)$ & \\
\hline FIGO stage & & & & $<0.001$ \\
\hline I & 25 & $20(92 \%)$ & $5(20 \%)$ & \\
\hline II & 21 & $13(52 \%)$ & $8(38 \%)$ & \\
\hline III & 89 & $36(40 \%)$ & $53(60 \%)$ & \\
\hline IV & 25 & $6(24 \%)$ & $19(76 \%)$ & \\
\hline
\end{tabular}



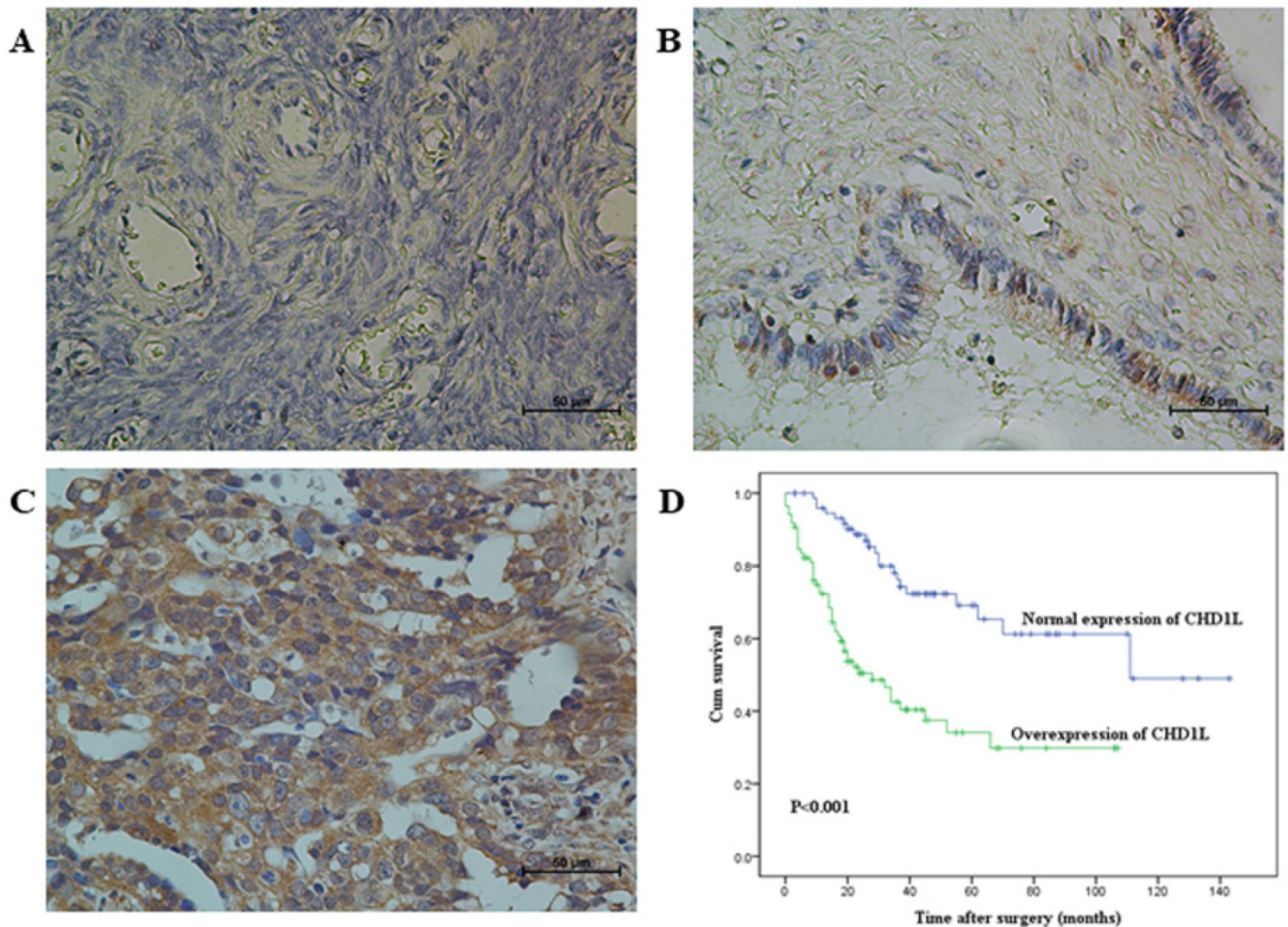

Figure 1. Immunohistochemical staining of CHDIL in human ovarian tissues and Kaplan-Meier survival analysis according to CHDIL expression in 160 EOC patients (log-rank test). A. Normal expression of CHDIL was observed in a normal surface epithelium of ovary (400x). B. An ovarian cystadenoma showed low expression of CHDIL, in which about $20 \%$ of tumor cells showed moderate positive staining of CHDIL (400x). C. Overexpression of CHDIL was detected in an ovarian carcinoma (case 93), in which more than $90 \%$ of carcinoma cells showed strong positive staining of CHDIL (400x). D. Probability of survival of patients: overexpression of CHDIL, $n=85$; normal expression of CHDIL, $n=75(P<0.001)$.

\section{Ectogenic overexpression of CHDIL transfected with plasmid promotes EOC cell invasion and metastasis in vitro}

Based on the five EOC cell lines analyzed, we found that HO8910 cells expressed relatively low levels of endogenous CHD1L protein, and on the contrary, ES2 and A2780 cells expressed relatively high levels of endogenous CHD1L protein (Fig. 2A). To evaluate the tumorigenic ability of CHD1L overexpression, HO8910-CHD1L cell lines that were transfected with plasmid [pcDNA3.1(+)-CHD1L] were used to perform an in vitro Matrigel invasion assay (Fig. 2B, left). We found that the invasive ability of the HO8910-CHD1L cells was significantly stronger than that of the control HO8910-vector cells in the Matrigel invasion assays ( $P<0.05$, Fig. 2B, right).

\section{Short hairpin RNA-mediated CHDIL silencing inhibits EOC cell invasion and metastasis in vitro}

To investigate the effects of CHD1L-silencing on invasion and metastasis, A2780 and ES2 cells were transfected with plasmids for either control shRNA or CHD1L-specific shRNAs that displayed the strongest effect in knocking down endogenous CHD1L expression in EOC cells (Fig. 2C, left). Silencing endogenous CHD1L expression by shRNA in A2780 and ES2 cells completely abolished the invasive ability of both the shCHD1L-A2780 and shCHD1L-ES2 cells in Matrigel invasion assays $(P<0.05$, Fig. $2 \mathrm{C}$, right).

\section{CHDIL up-regulates METAP2 expression in EOC cells}

To investigate any underlying downstream genes regulated by CHD1L expression, we extracted RNA of ES2-shControl and ES2-shCHD1L-1 cells, and detected the mRNA expression profiles using the Human Tumor Metastasis RT ${ }^{2}$ Profiler $^{\mathrm{TM}}$ PCR Array (Super Array Bioscience, America), which contains 84 cell invasion/metastasis-related genes. Examining the differential expression of these genes revealed that a total of 11 genes were differentially expressed by more than 2-fold in ES2-shCHD1L-1 cells. Eight genes 
were found to be downregulated (CD82, METAP2, NME4, RORB, CXCL12, TSHR, MMP11 and ITGA7) and three were found to be upregulated (TNFSF10, CDH11 and MMP3) (Fig. 3A). Next, we applied Western Blotting to analyzed protein expression levels of these genes. Consistent with the mRNA expression in the real-time PCR array, downregulated protein expression of CD82, METAP2 and NME4 was found in ES2-shCHD1L-1 cells, while upregulated protein expression of TNFSF10 was found in ES2-shCHD1L-1 cells (Fig. 3B).

\section{Correlation between the expression of METAP2 and that of CHD1L in EOC}

In order to further confirm whether CD82, METAP2, NME4 and TNFSF10 are potential downstream target genes regulated by $\mathrm{CHD} 1 \mathrm{~L}$, we quantified the correlations between the expression levels of these genes and that of CHD1L by IHC in our cohort of EOC tissue microarrays. In 153 of the 160 tissue samples, CHD1L and METAP2 were examined successfully and simultaneously by IHC (Fig. 3C). Our results show that the expression levels of CHD1L are significantly associated with METAP2 $(P<0.05$, Table 3). Interestingly, we found no significant correlation between the expression levels of CHD1L and CD82, NME4 or TNFSF10.

\section{Discussion}

Although the treatment of EOC has been improved greatly in recent years, the mortality rate is still very high [1]. The lack of any good biological markers for early detection or an accurate prognostic prediction is partly responsible for the high mortality of EOC. Our previous study demonstrated the presence of CHD1L overexpression in EOC and reported that overexpression of CHD1L protein is significantly correlated with the metastasis proceeding of ovarian carcinoma. We also reported that CHD1L protein expression, as examined by IHC, may act as a novel prognostic biomarker for patients with EOC [16]. In the present study, we increased our sample size and further confirmed that the degree of CHD1L overexpression increased sequentially from the normal ovarian tissues, to ovarian cystadenoma, to borderline tumors, and finally to EOC tissues.
A

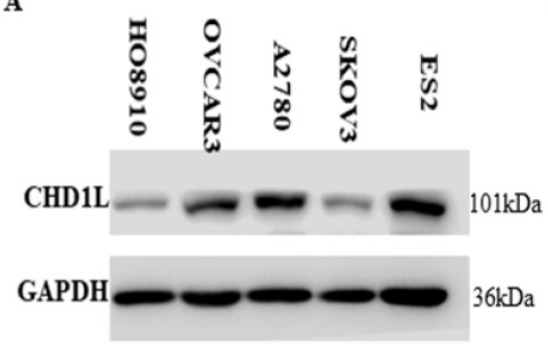

C

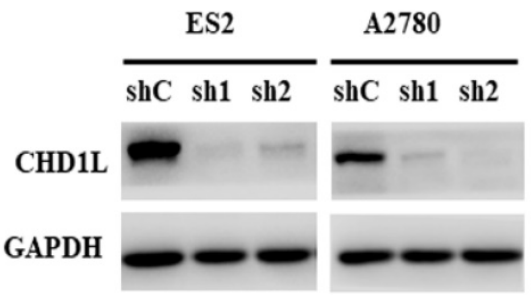

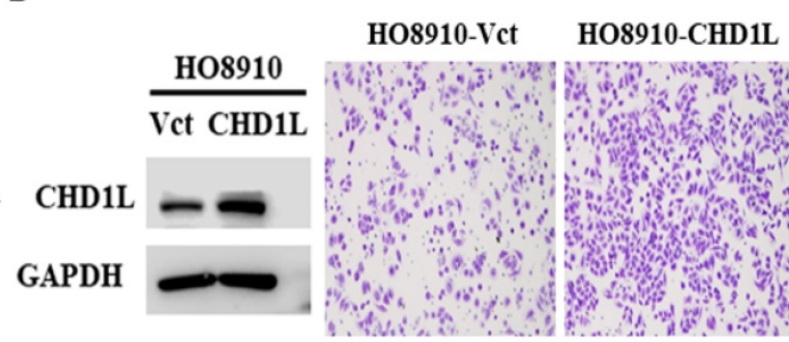
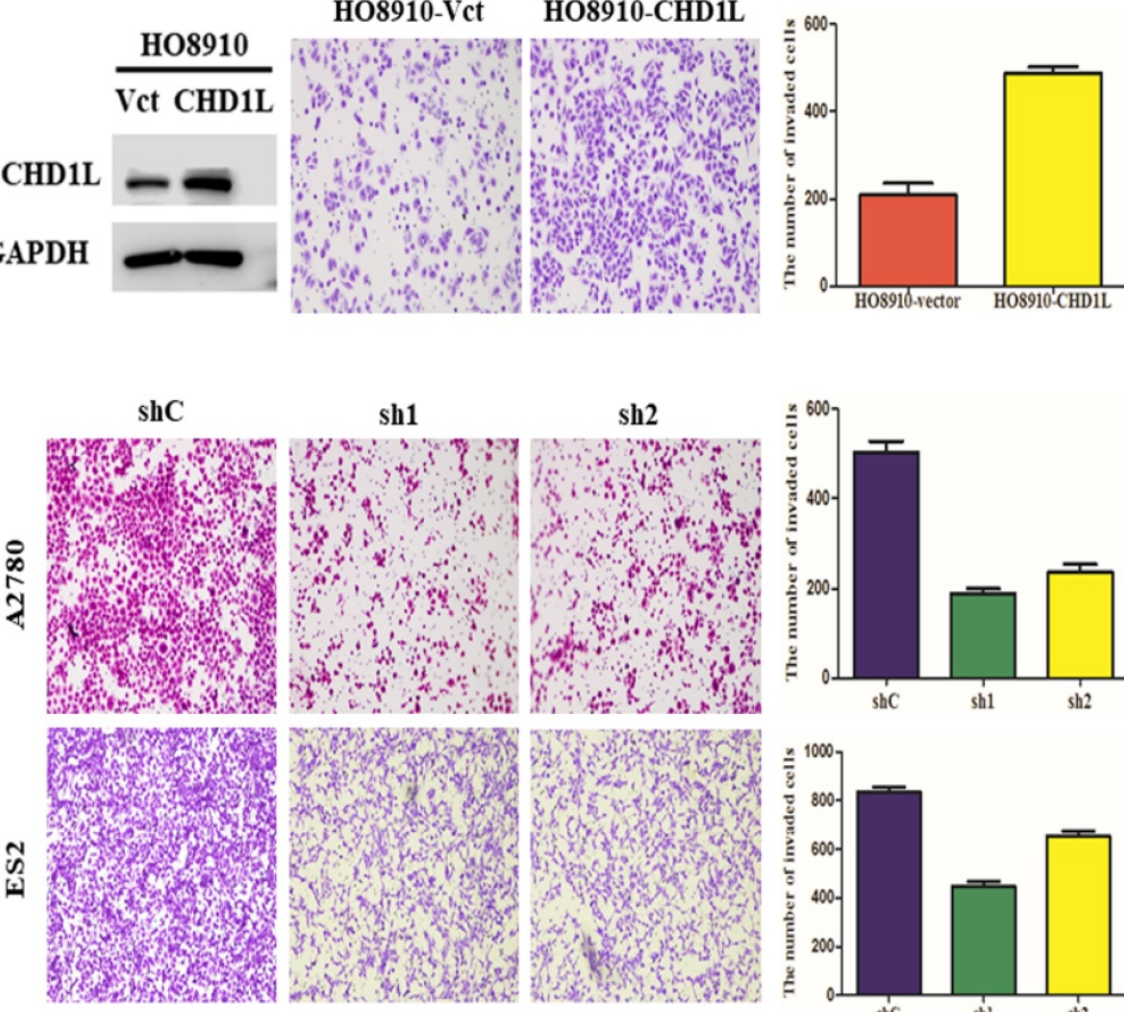

$\operatorname{sh} 1$

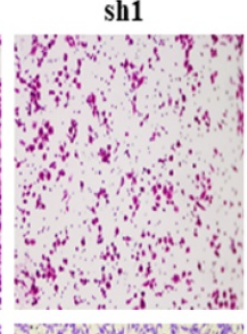

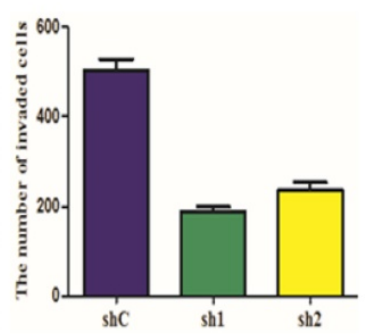
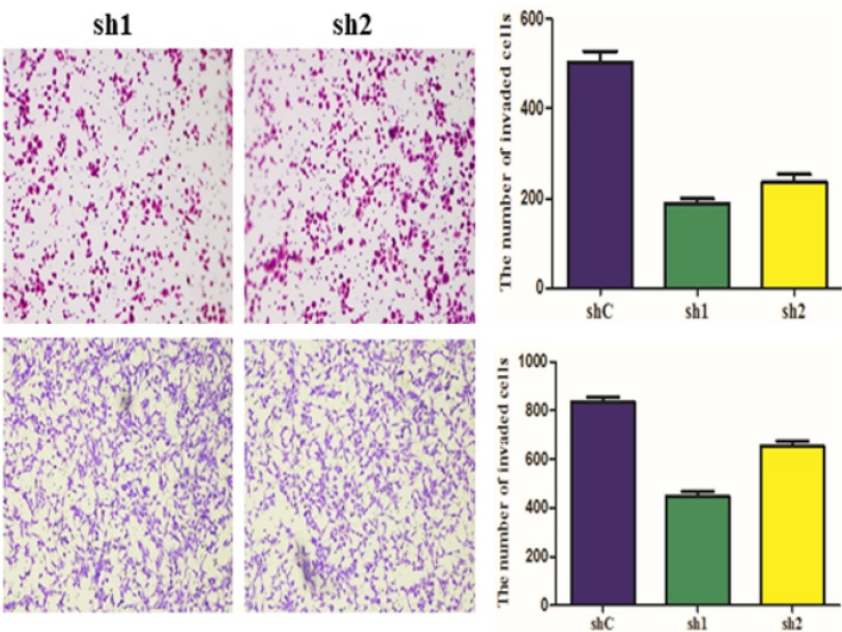

Figure 2. Overexpression of CHDIL enhances EOC cell invasion and silencing of CHDIL by RNA interference inhibits EOC cell invasion in vitro. A. The expression levels of CHDIL in 5 EOC cell lines by Western blotting analysis. B. Overexpression of CHDIL enhances EOC cell invasion in vitro. Left: Western blotting reveals that ectopic expression of CHDIL was substantially increased in HO8910-CHDIL cells compared with that in HO8910-vector cells. Right: Ectopic overexpression of CHD1L enhanced HO8910 cell invasion in a Transwell assay. The numbers of invaded cells in the HO8910-CHDIL and control groups are shown in the right panel. Data are the mean \pm standard error (SE) of three independent experiments; $P<0.05$ by Student's $t$ test. C. Silencing of CHDIL by RNA interference inhibits EOC cell invasion in vitro. Left: Western blotting reveals that CHDIL was efficiently knocked down by the treatment with CHDIL-shRNA1 and CHDIL-shRNA2. Right: Cell invasion was evaluated using a Matrigel invasion chamber. Silencing of CHD1L decreased ES2 and A2780 cells invasive capacity. The numbers of invaded cells in the shCHD1L and control groups are shown in the right panel. Data are the mean \pm standard error (SE) of three independent experiments; $P<0.05$ versus cells transfected with shC by Student's $t$-test. 
Furthermore, CHD1L overexpression is significantly associated with histological type and advanced $\mathrm{pT} / \mathrm{pN} / \mathrm{pM}$ status, as well as FIGO stage of EOC. Similar results have also been observed in human non-small-cell lung cancer, in which overexpression of CHD1L was reported to be associated with lymph node metastasis and/or distant organ metastasis [12]. These studies support our data showing that CHD1L plays an important role in the promotion of EOC cell invasion and metastasis.

To date, it is still not clear what the function of CHD1L is. In the present study, we evaluated CHD1L protein expression levels in six different human ovarian carcinoma cell lines (i.e. ES2, OVCAR-3, A2780, HO-8910 and SKOV3) using western blotting analysis. We found that HO8910 cell lines displayed relatively low levels of endogenous CHD1L protein expression, while on the contrary, the ES2 and A2780 cell lines displayed relatively high levels of endogenous CHD1L protein expression. Decreasing expression levels of CHD1L protein was accomplished using a specific lentiviral shRNA, and shRNA-mediated knockdown of endogenous CHD1L positively abolished the invasiveness and/or metastasis of A2780 and ES2 cells. Furthermore, ectogenic overexpression of CHD1L, transfected with plasmid, led to cell invasion and metastasis in vitro. These data provide evidence that CHD1L is involved in ovarian carcinoma cell invasion and/or metastasis and that overexpression of CHD1L may be a critical factor in promoting EOC cell metastasis.

Table 3. Association of CHDIL expression with METAP2 in 153 EOC

\begin{tabular}{lllll}
\hline Variable & \multirow{2}{*}{$\begin{array}{l}\text { All } \\
\text { cases }\end{array}$} & \multicolumn{3}{l}{ CHD1L protein } \\
\cline { 3 - 5 } & & & \\
\hline METAP2 & & & \\
Low-level expression & 74 & $42(57 \%)$ & $32(43 \%)$ & 0.020 \\
High-level expression & 79 & $30(38 \%)$ & $49(62 \%)$ & \\
\hline
\end{tabular}

a Chi-square test.

However, despite there being a clear connection between CHD1L and cell invasion/metastasis, the precise molecular mechanisms by which CHD1L promotes cancer cell invasion/metastasis are still unclear. A previous study demonstrated that CHD1L upregulates the expression of ARHGEF9, which subsequently activates Cdc42, causing filopodia
A

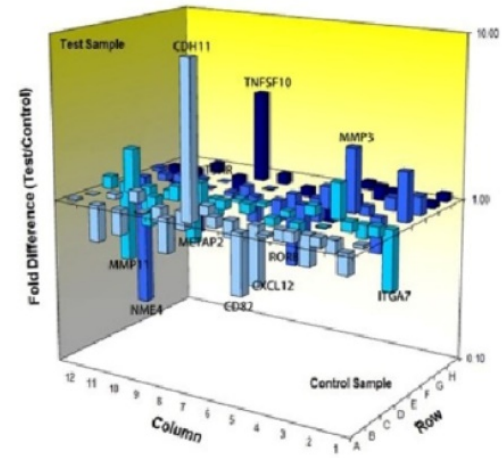

C
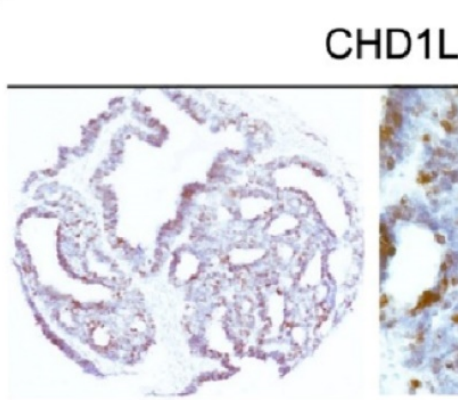

$X 100$

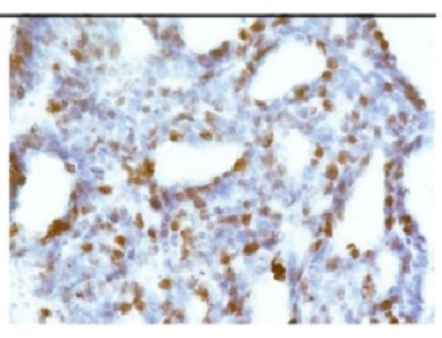

$\times 400$
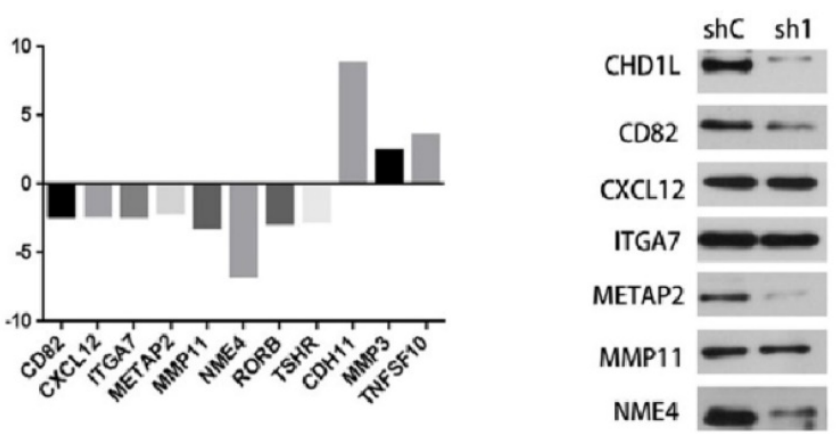

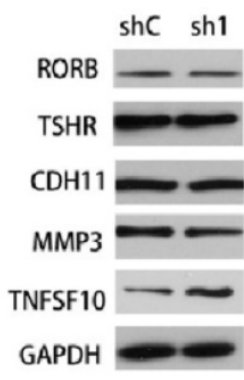

Figure 3. The associations of CHDIL and METAP2 expression in EOC cells. A. A total of 11 genes were differentially expressed by more than 2-fold in ES2-shCHDIL-1 cells compared with that in ES2-shControl cells. Eight genes were found to be downregulated (CD82, METAP2, NME4, RORB, CXCL12, TSHR, MMP11 and ITGA7) and another three genes were found to be upregulated (TNFSF10, CDH11 and MMP3), as detected using the Human Tumor Metastasis RT ${ }^{2}$ Profiler TM PCR Array (Super Array Bioscience, America). B. Silencing of CHD1L by shRNA down-regulated CD82, METAP2 and NME4 expression in ES2-shCHD1L-1 cells, while up-regulated TNFSF10 expression in ES2-shCHDIL-1 cell, as detected by Western blotting. C. The associations of CHDIL and METAP2 expression in EOC patients. Overexpression of CHD1L and high-level expression of METAP2 were detected by IHC in an ovarian carcinoma (case 140), in which more than $80 \%$ of carcinoma cells showed strong positive staining of these proteins. 
formation, epithelial-mesenchymal transition (EMT), and finally promotes hepatocellular carcinoma cell invasion and metastasis [19]. Li et al. [20] recently demonstrated that CHD1L activates expression of SPOCK1, which activates Akt signaling to then block apoptosis and promote hepatocellular carcinoma cell invasiveness and metastasis in mice. However, little is known about the underlying mechanisms involved in CHD1L-regulated EOC cell invasion and metastasis. To further investigate the downstream molecular events involving CHD1L-regulated invasiveness and metastasis in EOC, we compared mRNA expression profiles between ES2-shCHD1L-1 cells and ES2-shControl cells using a Human Tumor Metastasis real-time PCR array, which contains 84 well-known cell invasion/metastasis-related genes. Of the 84 genes, we identified a total of 11 genes that were differentially expressed by more than 2-fold, including eight genes that were downregulated (CD82, METAP2, NME4, RORB, CXCL12, TSHR, MMP11 and ITGA7) and three genes that were upregulated (TNFSF10, CDH11 and MMP3). Next, protein expression of these differentially regulated genes in the ES2-shCHD1L-1 cells was quantified via western blot assay. Consistent with the mRNA expression data, we found that CD82, METAP2 and NME4 were downregulated and TNFSF10 was upregulated. To further confirm these results, the expression status of these genes (CD82, METAP2, NME4 and TNFSF10) was detected using IHC in our TMA assay. Further correlation analysis revealed that there was no significant correlation between the expression levels of CHD1L and that of CD82, NME4 and TNFSF10, regardless of the cut off value used. Interestingly, we did observe a significant positive correlation between overexpression of CHD1L and METAP2 expression levels. Given this, it is reasonable to propose that $\mathrm{CHD} 1 \mathrm{~L}$ promotes cell invasion via the regulation of METAP2 expression in our EOC cells.

METAP2 (methionyl aminopeptidase 2) is a glycoprotein that is responsible for the processing of the N-terminal initiator methionine from nascent proteins in cells [21]. Upregulated expression of METAP2 promotes cell proliferation [22], while silencing METAP2 using siRNA induces significant inhibition of the proliferation of human umbilical vein endothelial cells $[23,24]$. It has been reported that overexpression of METAP2 is observed in various samples of human malignancies, such as breast cancer, colon cancer, lung cancer, ovarian cancer and prostate carcinomas and promotes the development of tumors [25]. A recent study demonstrated that regarding non-small-cell lung cancer (NSCLC), that METAP2 is involved in regulating cell proliferation and apoptosis in NSCLC. Furthermore, METAP2 inhibition may provide prevention of tumor cell growth, development and progression in NSCLC patients [26]. However, there is no evidence indicating METAP2 involvement being directly correlated with metastatic potential in cancer, but it has been demonstrated that METAP2 is a new target for the metastasis-associated protein, S100A4 [27]. Taken in the context of our results here on the function of CHD1L in EOC cells, these observations indicate that METAP2 might be a potential downstream target in the aggressive behavior of CHD1L-mediated EOC, and as a result promotes cancer cell invasion and metastasis. Further studies are obviously needed to elucidate the precise molecular mechanisms of $\mathrm{CHD} 1 \mathrm{~L}$ regulation of METAP2 in EOC. Furthermore, it may lead to a more effective management of EOC progression, via precise prognostication and treatment targeted at these molecules.

Here based on our previous study and the present study, we show that high expression of CHD1L plays a potential role in the inducement of EOC cancer cell invasion and/or metastasis via the regulation of METAP2 expression. Furthermore we showed that silencing CHD1L, through inhibition of CHD1L expression levels may be a promising therapeutic strategy for prevention and intervention of metastasis of EOC.

\section{Materials and Methods}

\section{Patients and tissue microarray}

In this study, paraffin-embedded pathological specimens from 160 patients diagnosed with EOC and treated between 2003 and 2010 were obtained from the archives of the Department of Pathology, the First Affiliated Hospital, Sun Yat-Sen University, Guangzhou, China, and prepared for ovarian tissue microarray (TMA). The patients were selected based on availability of resection tissue, as well as follow-up data. Any patients who had accepted preoperative radiation or chemotherapy were excluded from the study. In addition, patients that died from unknown causes or emergency were also excluded from this study. The TMA was constructed according to a method described previously [17]. Prior patient's informed consent and the Institute Research Medical Ethics Committee of Sun Yat-Sen University granted approval were obtained for the research purposes of this study.

\section{Immunohistochemistry staining}

The immunohistochemical studies were carried out using a standard streptavidin-biotin-peroxidase complex method demonstrated previously [18]. Briefly, tissue sections were deparaffinized and 
rehydrated. The endogenous peroxidase activity of the tissue was blocked using 3\% hydrogen peroxide for $10 \mathrm{~min}$. For antigen retrieval, slides were immersed and boiled in $10 \mathrm{mM}$ citrate buffer ( $\mathrm{pH}$ 6.0) for $15 \mathrm{~min}$ in a pressure cooker, and then non-specific binding was blocked by $5 \%$ normal goat serum for 10 min. The slides were incubated with anti-CHD1L (1:100 dilution; Abcam), a 1:200 dilution of monoclonal antibody against cluster of differentiation 82 (CD82), non-metastatic cells 4 (NME4), tumor necrosis factor (ligand) superfamily member 10 (TNFSF10) or methionyl aminopeptidase 2 (METAP2) at $4^{\circ} \mathrm{C}$ overnight in a moist chamber. The slides were sequentially incubated with biotinylated goat antimouse IgG (Santa Cruz Biotechnology) at a dilution of 1:100 and then allowed to react with the streptavidinperoxidase conjugate, each for $30 \mathrm{~min}$ at room temperature. Staining was developed using the 3, 5-diaminobenzidine (DAB) Substrate Kit (Dako) according to the manufacturer's instructions, followed by Mayer hematoxylin counterstaining.

\section{EOC cell lines and culture conditions}

The EOC cell lines ES2, HO8910 and SKOV3 were maintained in RPMI-1640 medium, and the EOC cell lines A2780 and OVCAR3 were cultured in DMEM medium, both of which supplemented with $10 \%$ fetal bovine serum (FBS) (GIBCO) and 1\% antibiotics $(100 \mathrm{U} / \mathrm{mL}$ penicillin and $100 \mu \mathrm{g} / \mathrm{mL}$ streptomycin).

\section{Protein extraction and Western blotting}

Protein was extracted from the EOC cells by Radio-Immunoprecipitation Assay (RIPA) Lysis Buffer (Beyotime) at $4{ }^{\circ} \mathrm{C}$. Protein concentrations were detected using the Bicinchoninic Acid (BCA) Protein Assay Kit (BioRad, Hercules, CA, USA). Equal amounts of whole-cell lysates were separated by SDS-polyacrylamide gel electrophoresis and transferred onto a polyvinylidene difluoride (PVDF) membrane (Millipore, Danvers, MA, USA) and then incubated with primary mouse monoclonal antibodies against the following human proteins: CHD1L (1:1000 dilution), METAP2 (1:1000 dilution), TNFSF10 (1:1000 dilution), CD82 (1:1000 dilution), and NME4 (1:1000 dilution) (BD Transduction Laboratories) overnight at $4{ }^{\circ} \mathrm{C}$. GAPDH was used as a loading control (1:5000 dilution, BD Transduction Laboratories). After the PVDF membranes were incubated with the secondary antibody (goat antimouse, 1:10,000 dilution, Cell Signaling Technology, Danvers, MA, USA), the immunoreactive proteins were visualized by enhanced chemiluminescence detection reagents (Amersham Biosciences, Uppsala,
Sweden) in accordance with the manufacturer's instructions.

\section{Plasmid Constructs and Transfection}

To evaluate the tumorigenic ability of CHD1L, the full-length CHD1L cDNA was amplified by PCR and cloned into a pcDNA3.1+ expression vector (Invitrogen) as described previously [8]. The expression plasmids were transfected into HO9810 cells using Lipofectamine 2000 (Invitrogen) according to the manufacturer's instructions. Stable CHD1L expressing clones were selected using Geneticin (Roche), and the level of CHD1L expression was detected by Western blot analysis. Cells transfected with empty vector were used as controls.

\section{Knockdown of CHDIL by lentiviral short hairpin RNA (shRNA)}

Based on the CHD1L sequence, we constructed a specific lentiviral shRNA for CHD1L using the sequences of shRNA1 (5'-GCC AAG AGA AGG AGA-3') and shRNA2 (5'-CGT ATT GGA CAT GCC ACG AAA-3'), which have been previously verified to efficiently knockdown endogenous CHD1L expression in human cancer cells [19]. The lentiviral particles were collected, filtered, and reserved $72 \mathrm{~h}$ post transfection. Next, we transfected the EOC cell lines with the lentivirus particles, each transfection was performed in triplicate and repeated three times. Cells were harvested $72 \mathrm{~h}$ post transfection and knockdown efficiency was quantified using Western blotting.

\section{Cell invasion assay}

Post-second infection, cells were seeded onto a synthetic basement membrane (Falcon TM Cell Culture Inserts, BD, Oxford) located at the bottom of the wells in a 24-well culture plate. In our invasion assay, polycarbonate filters ( $8 \mu \mathrm{m}$ pore size) were coated with $20 \mu \mathrm{g}$ Matrigel and placed in a modified Boyden chamber. Fetal bovine serum was added to the lower chamber as a chemoattractant. Cells were then incubated at $37^{\circ} \mathrm{C}$ and allowed to invade through the Matrigel barrier for either 18 or $36 \mathrm{~h}$. After the incubation period, non-invading cells on the upper filter were cleaned up by a cotton swab and invading cells on the bottom of the filter were fixed and stained with crystal violet. The invasive cells were then quantified in five fields under an inverted microscope. Experiments were performed in triplicate with a minimum of 40 grids ( $\times 400$ magnification) per filter counted.

\section{Real-time polymerase chain reaction gene array}

Post the second dose of shRNA knockdown 
experiments, we extracted RNA from shCHD1L and shC using Trizol (Invitrogen) and cleared them by RNeasy ${ }^{\circledR}$ Min Elute ${ }^{\mathrm{TM}}$ Cleanup Kit (Qiagen, Valencia, CA). Total RNA was reverse transcribed through Super-Script III Reverse Transcriptase (Invitrogen) and polymerase chain reaction (PCR) was used to amplify complementary DNA using the $2 \times$ Super Array PCR master mix (Super Array Bioscience, Frederick, MD). An Opticon ${ }^{\mathrm{TM}}$ DNA Engine ABI PRISM7900 system (Applied Biosystems, Foster City, CA) was used for Real-time PCR on each sample using the Human Tumor Metastasis RT2 ProfilerTM PCR Array (Super Array Bioscience), following the manufacturer's instructions. Finally, data was normalized to GAPDH levels using the $\Delta \Delta \mathrm{Ct}$ method.

\section{Statistical analyses}

The SPSS statistical software package (SPSS Standard version 20.0, SPSS Inc. Chicago, IL) was used for statistical analysis. Data are presented as the mean \pm standard deviation (SD) of 3 independent experiments. The independent Student's $t$ test was performed to compare the invasive ability between any 2 pre-selected groups. The association of CHD1L expression with clinicopathologic features of EOC patients and the correlation between CHD1L expression and METAP2 expression in the EOC clinical samples were evaluated using the $\chi^{2}$ test. Survival curves were obtained through the KaplanMeier method. Log-rank test was used to compare different survival curves. $P$ values less than 0.05 were considered to be statistically significant.

\section{Acknowledgements}

This study was supported by grants from the Nature Science Foundation of China (No.81772769), and from the Research Project in the Science and Technology Bureau in Guangzhou (No. 201704020125), and from Guangdong Basic and Applied Basic Research Foundation (No. 2019A1515011610 and No. 2020A1515010169).

This manuscript has been thoroughly edited by a native English speaker from an editing company. Editing Certificate will be provided upon request.

\section{Availability of data and materials}

The datasets supporting the conclusions of this article are included within the article.

\section{Authors' contributions}

WPH, YYG and GPY carried out the experimental approach and drafted the manuscript. HLL and TTS evaluated the clinical records and helped to draft the manuscript. YZ, LMT and XHL participated in carrying out the experimental approach. ZWZ and LLOY participated in the statistical analysis and participated in its coordination. WPH, ZSY, DX and GFY participated in the design of the study and coordination and wrote the manuscript. All authors read and approved the final manuscript.

\section{Competing Interests}

The authors have declared that no competing interest exists.

\section{References}

1. Siegel RL, Miller KD, Jemal A. Cancer statistics, 2018. CA Cancer J Clin. 2018; 68(1):7-30.

2. Desai A, Xu J, Aysola K, et al. Epithelial ovarian cancer: An overview. World J Transl Med 2014; 3(1):1-8.

3. Survival rates for ovarian cancer, by stage. 2014, American Cancer Society.

4. Vaughan S, Coward JI, Bast RC Jr, et al. Rethinking ovarian cancer: recommendations for improving outcomes. Nat Rev Cancer 2011; 11:719-725.

5. Jayson GC, Kohn EC, Kitchener HC, et al. Ovarian cancer. Lancet 2014; 384(9951):1376-88.

6. Chen L, Chan TH, Guan XY. Chromosome 1q21 amplification and oncogenes in hepatocellular carcinoma. Acta Pharmacol Sin 2010; 31(9):1165-71.

7. Flaus A, Martin DM, Barton GJ, et al. Identification of multiple distinct Snf2 subfamilies with conserved structural motifs. Nucleic Acids Res 2006; 34(10): 2887-905.

8. Ma NF, Hu L, Fung JM, et al. Isolation and characterization of a novel oncogene, amplified in liver cancer 1, within a commonly amplified region at 1q21 in hepatocellular carcinoma. Hepatology 2008; 47(2):503-10.

9. Wu J, Zong Y, Fei X, et al. Presence of CHD1L over-expression is associated with aggressive tumor biology and is a novel prognostic biomarker for patient survival in human breast cancer. PLoS One 2014; 9(8):e98673.

10. Su Z, Zhao J, Xian G, et al. CHD1L is a novel independent prognostic factor for gastric cancer. Clin Transl Oncol 2014; 16(8): 702-7.

11. Su FR, Ding JH, Bo L, et al. Chromodomain helicase/ATPase DNA binding protein 1-like protein expression predicts poor prognosis in nasopharyngeal carcinoma. Exp Ther Med 2014; 8(6):1745-1750.

12. He LR, Ma NF, Chen JW, et al. Overexpression of CHD1L is positively associated with metastasis of lung adenocarcinoma and predicts patients poor survival. Oncotarget 2015; 6(31):31181-90.

13. Mu QJ, Li HL, Yao Y, et al. Chromodomain Helicase/ATPase DNA-Binding Protein 1-Like Gene (CHD1L) Expression and Implications for Invasion and Metastasis of Breast Cancer. PLoS One 2015; 10(11):e 0143030

14. Liu C, Fu X, Zhong Z, et al. CHD1L Expression Increases Tumor Progression and Acts as a Predictive Biomarker for Poor Prognosis in Pancreatic Cancer. Dig Dis Sci 2017; 62(9):2376-2385.

15. Liu ZH, Zhang Q, Ding YJ, et al. Overexpression of CHD1L is associated with poor survival and aggressive tumor biology in esophageal carcinoma. Oncotarget 2017; 8(43):74178-74187.

16. He WP, Zhou J, Cai MY, et al. CHD1L protein is overexpressed in human ovarian carcinomas and is a novel predictive biomarker for patients survival. BMC Cancer 2012; 12:437.

17. Xie D, Sham JST, Zeng WF, et al. Heterogeneous expression and association of $\beta$-catenin, p16 and c-myc in multistage colorectal tumorigenesis and progression detected by tissue microarray. Int J Cancer 2003; 107:896-902.

18. Chen L, Chan TH, Yuan YF, et al. CHD1L promotes hepatocellular carcinoma progression and metastasis in mice and is associated with these processes in human patients. J Clin Invest 2010; 120(4):1178-91.

19. Chen L, Yuan YF, Li Y, et al. Clinical significance of CHD1L in hepatocellular carcinoma and therapeutic potentials of virus-mediated CHD1L depletion. Gut 2011; 60:534-543

20. Yan Li, Lei-Lei Chen, Tim Hon Man Chan, et al. SPOCK1 Is Regulated by CHD1L and Blocks Apoptosis and Promotes HCC Cell Invasiveness and Metastasis in Mice. GASTROENTEROLOGY 2013; 144:179-191.

21. Datta B. MAPs and POEP of the roads from prokaryotic to eukaryotic kingdoms. Biochimie 2000; 82(2):0-107.

22. Wang J, Lou P, Henkin J. Selective inhibition of endothelial cell proliferation by fumagillin is not due to differential expression of methionine aminopeptidases. J Cell Biochem 2000; 77:465-473.

23. Bernier SG, Taghizadeh N, Thompson CD, et al. Methionine aminopeptidases type I and type II are essential to control cell proliferation. J Cell Biochem 2005; 95:1191-1203.

24. Yeh JR, Ju R, Brdlik CM, et al. Targeted gene disruption of methionine aminopeptidase 2 results in an embryonic gastrulation defect and endothelial cell growth arrest. Proc Natl Acad Sci USA 2006; 103:10379-10384.

25. Tucker LA, Zhang Q, Sheppard GS, et al. Ectopic expression of methionine aminopeptidase- 2 causes cell transformation and stimulates proliferation. Oncogene 2008; 27(28):3967-76. 
26. Shimizu H, Yamagishi S, Chiba H, et al. Methionine Aminopeptidase 2 as a Potential Therapeutic Target for Human Non-Small-Cell Lung Cancers. Adv Clin Exp Med 2016; 25(1):117-28.

27. Hideya Endo, Keizo Takenaga, et al. Methionine aminopeptidase 2 is a new target for the metastasis-associated protein, S100A4. J Biol Chem 2002; 277(29):2. 